\title{
Agir plutôt que réagir
}

Bruno Kesseli

Dr méd. et lic. phil, rédacteur en chef

L'assemblée plénière 2017 de l'Institut suisse pour la formation médicale postgraduée et continue (ISFM) a été marquée par la densité des informations échangées au cours d'intenses discussions et d'exposés intéressants. Tous les participants se sont accordés à dire que le corps médical devait aborder activement les défis à venir dans le domaine de la formation médicale avant que les décisions ne soient prises «ailleurs».

Après le message de bienvenue adressé aux délégués et aux hôtes, le président de l'ISFM, Werner Bauer, qui dirigeait l'assemblée plénière, a rendu hommage à Christina Kuhn. En tant que présidente et responsable de la Section formation universitaire de la Commission des professions médicales (MEBEKO), elle a assisté pendant plusieurs années aux séances de l'ISFM. Werner Bauer a relevé qu'elle a su assurer le lien entre le corps médical et l'administration de façon exemplaire. D'une part, grâce à son remarquable talent diplomatique, mais aussi par sa détermination et sa capacité à s'imposer. Le président de l'ISFM a terminé son éloge en la remer-

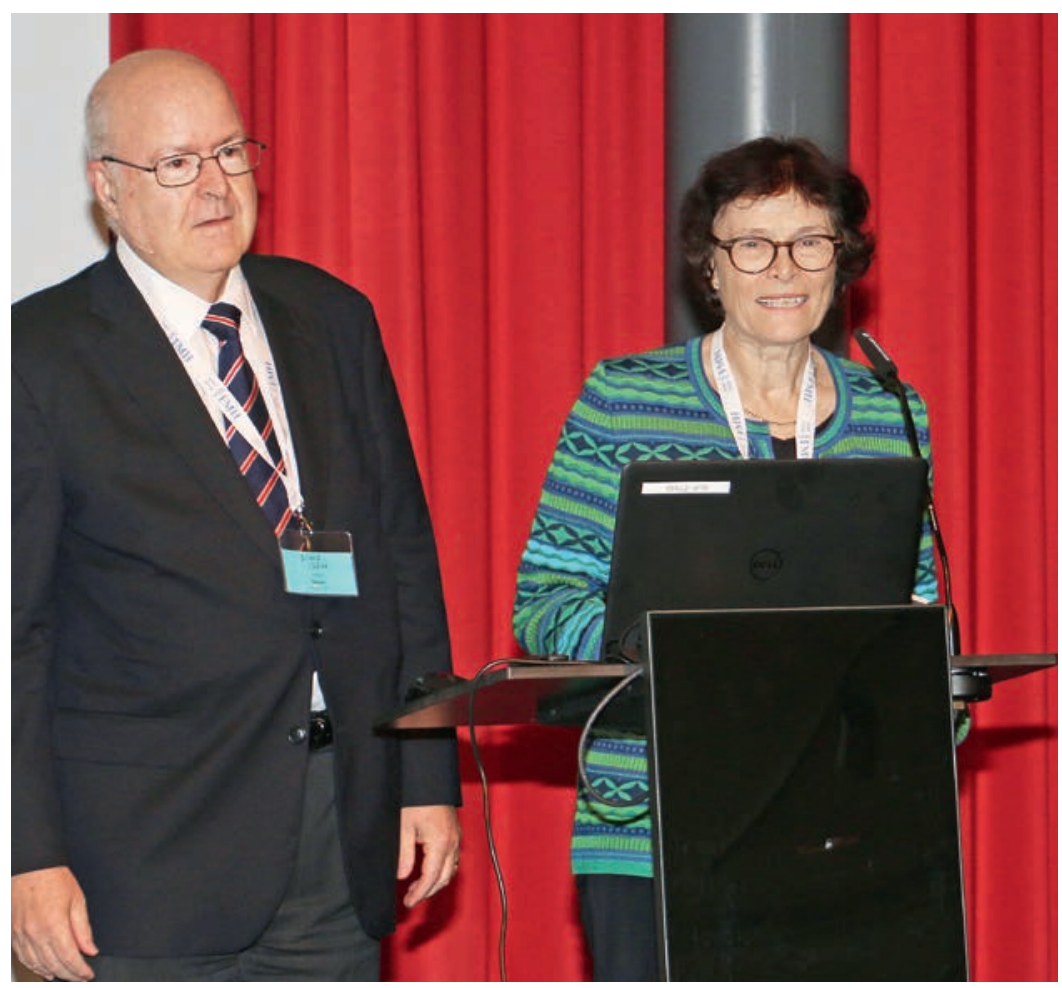

Christina Kuhn s'adresse au public après avoir reçu un hommage chaleureux du président de I'ISFM, Werner Bauer. ciant pour son «travail remarquable» et en lui remettant un petit cadeau.

\section{Infoflash}

Une fois de plus, l'infoflash a livré un bon aperçu de la diversité des activités de l'ISFM. Werner Bauer a donné des informations concernant l'accréditation des cursus de formation par le Département fédéral de l'intérieur qui bat son plein et devrait se terminer en été 2018 avec la publication des décisions d'accréditation. L'ISFM reste actif dans la plate-forme "Avenir de la formation médicale» lancée en 2010 par l'Office fédéral de la santé publique (OFSP) et la Conférence suisse des directrices et directeurs cantonaux de la santé (CDS). Les groupes formés sur les thèmes suivants tentent de trouver des réponses aux défis et problèmes actuels de la formation médicale:

- Financement de la formation postgrade des médecins

- Harmonisation des formations universitaire et postgrade

- Interprofessionnalité dans la formation médicale

- Relève pour la recherche clinique en Suisse

- Coordination de la formation postgrade des médecins

- Spécialisation croissante en médecine humaine du point de vue de l'organisation des hôpitaux

Le président a également fourni un aperçu des «chantiers» de l'ISFM. Le futur statut de la médecine d'urgence hospitalière et de la neuroradiologie est en cours de discussion avec des clarifications et entretiens à différents niveaux. Il est prévu d'établir une attestation de formation complémentaire pour les experts médicaux. Pour les domaines prévention et contrôle des infections (mieux connu sous le nom d'hygiène hospitalière) et chirurgie 
de la colonne vertébrale, il est également prévu de créer des qualifications spécifiques dans un proche avenir. Des formations approfondies sont attendues en chirurgie pédiatrique et en cardiologie (par ex. cardiologie interventionnelle et rythmologie). Werner Bauer a terminé ses explications en donnant des informations sur l'Union européenne des médecins spécialistes (UEMS) qui élabore des programmes de formation postgraduée européens (training requirements) dans ses sections et qui exerce de ce fait une influence croissante sur les programmes de formation postgraduée nationaux.

Christoph Hänggeli, directeur de l'ISFM, a résumé pour le plénum les principaux points de la révision de la loi sur les professions médicales et de ses ordonnances. La version révisée de la loi [1], qui est entrée en vigueur le $1^{\text {er }}$ janvier 2018, prévoit notamment que les médecins ne peuvent exercer leur profession en Suisse que s'ils sont inscrits au registre des professions médicales. Désormais, les médecins salariés doivent également disposer d'une autorisation de pratiquer cantonale s'ils exercent à titre d'activité économique privée, sous propre responsabilité professionnelle (par ex. dans un cabinet de groupe). De plus, la loi exige que les médecins doivent disposer des connaissances linguistiques nécessaires à l'exercice de leur profession.

\section{Une lueur d'espoir budgétaire}

Le budget 2018 a été approuvé par la Chambre médicale en octobre 2017. Comme les années précédentes, il prévoit un déficit qui devrait cependant rester en deçà des exercices précédents (-609000). Un déficit est encore attendu pour 2019, mais l'équilibre devrait être rétabli à partir de 2020. Du côté des recettes, le nombre de titres de spécialiste délivrés est un facteur important. En 2016, lorsque l'exercice a été clôturé sur une perte de 1,25 million de francs, le total des titres de spécialiste délivrés était inférieur de 30\% à l'année précédente, ce qui ne correspond toutefois pas à la tendance générale. En ce qui concerne les dépenses 2018, 400000 francs sont prévus pour l'accréditation et 500000 francs pour le développement du nouveau logbook électronique. Comme la fortune se monte actuellement encore à 2,6 millions de francs, l'ISFM renonce pour l'instant à une augmentation des émoluments perçus pour les titres.

\section{Le logbook électronique, un projet complexe}

Le logbook électronique permet aux médecins d'inscrire les différentes étapes de leur formation postgra-

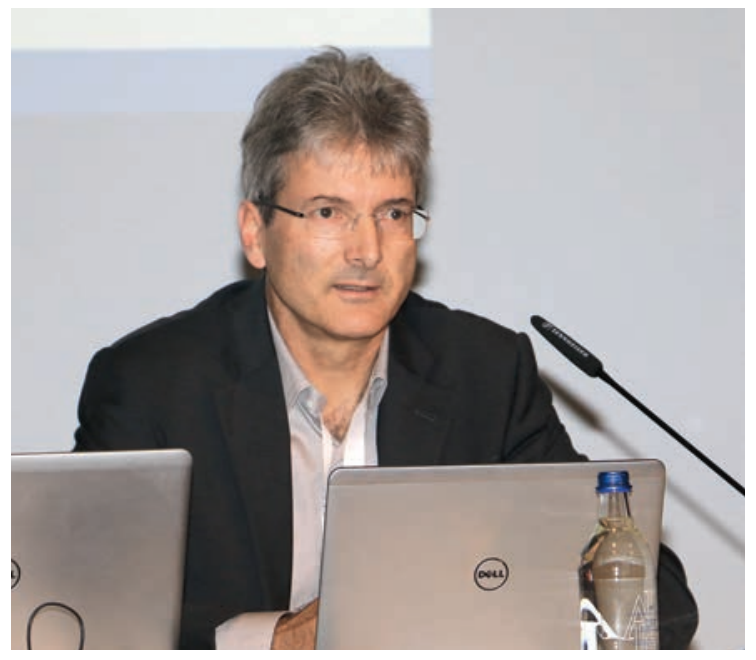

Christoph Hänggeli, directeur de I'ISFM, évoque notamment la loi sur les professions médicales révisée et le budget 2018.

duée. Les informations qu'il renferme servent de base à toute demande en vue de l'octroi d'un titre de spécialiste. Les problèmes de fond inhérents à ce projet informatique complexe ont rendu inévitable le développement d'un nouveau logbook électronique. Comme Werner Bauer et Christoph Hänggeli l'ont souligné, les travaux sont actuellement en cours et étroitement accompagnés par un expert externe, qui donne de bonnes notes à l'équipe de projet, mais qui met en garde contre une surcharge du projet. L'informaticien médical responsable du développement, Lukas Wyss, a présenté les éléments clés du nouveau produit censé associer fiabilité, convivialité, possibilités de développement et facilité d'entretien. L'intégration des catalogues des objectifs de formation des différentes disciplines, qui présentent des structures très hétérogènes, reste toutefois un point critique. Répondant à une question du public, Lukas Wyss a précisé qu'un objectif du projet est de pouvoir éviter d'adapter les programmes de formation postgraduée existants.

Dans le vote qui a suivi, le plénum a approuvé les propositions suivantes (avec trois abstentions):

- Les objectifs de formation qui sont exigés dans les différents programmes de formation postgraduée peuvent être atteints dans tous les établissements de formation postgraduée disposant de la reconnaissance correspondante (le système du logbook électronique ne permet pas de contrôler la reconnaissance ou la plausibilité des sessions de formation).

- SNOMED CT (version internationale) sert de nomenclature de référence - d'autres classifications (par ex. $\mathrm{CHOP}, \mathrm{AO}$ ) peuvent être ajoutées ultérieurement par les sociétés de discipline. 


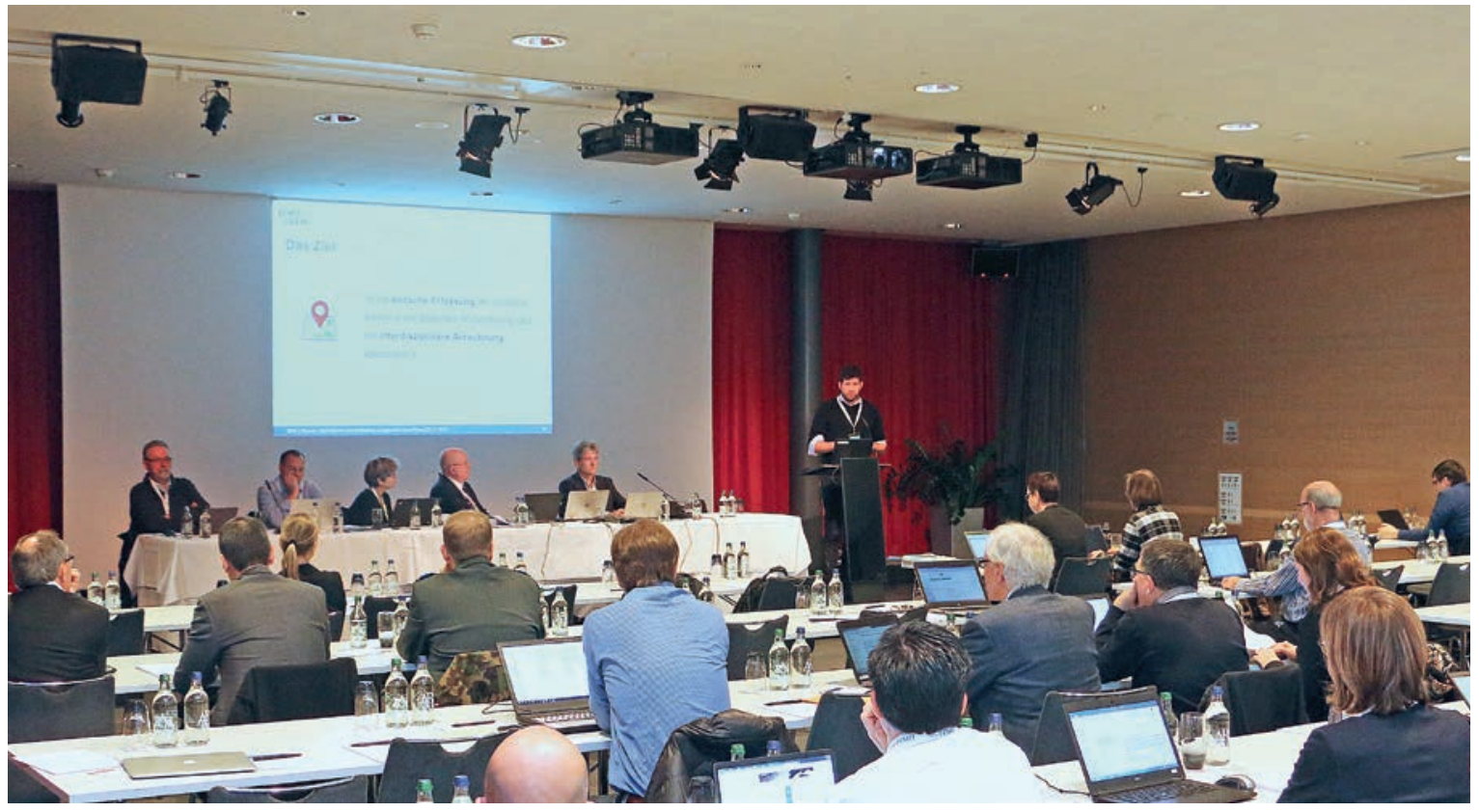

La direction de I'ISFM et le plénum suivent les explications de Lukas Wyss au sujet du logbook électronique.

- Le paramétrage du programme de formation postgraduée dans le logbook électronique doit, d'une part, être confirmé par la présidence et le responsable de la formation postgraduée de la société de discipline et, d'autre part, par la Commission des titres de l'ISFM.

\section{Le pilotage de la formation postgraduée suscite le scepticisme}

Bernadette Sütterlin de l'EPF Zurich a présenté certains des résultats de l'enquête annuelle consacrée à la formation postgraduée. Ce faisant, elle s'est focalisée sur les trois domaines suivants abordés dans des questions modulaires:

- Pilotage de la formation postgraduée par le nombre de postes de formation

- Moment du choix de la discipline

- Compatibilité entre formation postgraduée et vie privée

Les questions des deux premiers modules ont également été soumises aux responsables des établissements de formation postgraduée. Parmi eux, 52\% se sont déclarés défavorables à un pilotage ciblé du nombre de médecins formés par discipline. $40 \%$ approuveraient un pilotage et $8 \%$ sont indécis. A l'inverse, seuls 32\% des médecins en formation postgraduée rejettent un pilotage, alors que $38 \%$ se déclarent favorables. Avec 30\%, la part des indécis est comparativement élevée dans ce groupe. Les deux groupes sont d'accord sur le fait que le choix de la discipline de la formation postgraduée ne doit pas se faire pendant les études (taux de refus d'en- viron $80 \%$ ). Plus de $30 \%$ sont d'avis que l'on peut raisonnablement attendre des médecins qu'ils prennent cette décision durant la deuxième année de formation postgraduée, alors qu'au maximum $21 \%$ des personnes interrogées citent les autres années de formation postgraduée. Les médecins en formation postgraduée qui travaillent à temps partiel estiment que la compatibilité entre vie professionnelle et vie privée est nettement meilleure que chez leurs collègues travaillant à plein temps. A leur avis, le principal potentiel pour mieux concilier vie professionnelle et vie privée réside dans l'allègement des tâches administratives.

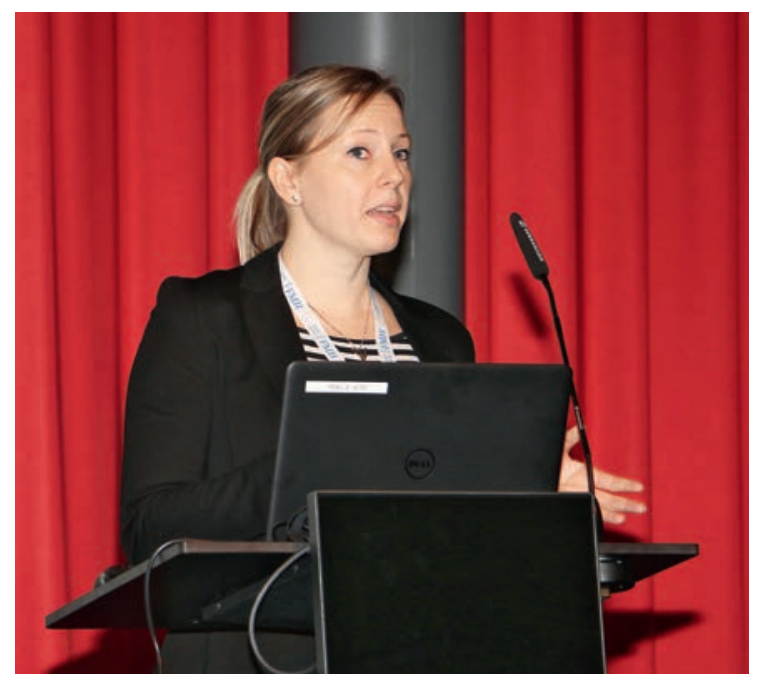

Bernadette Sütterlin de I'EPF Zurich présente certains des résultats de I'enquête 2017 consacrée à la formation médicale postgraduée. 


\section{La thèse de doctorat a valeur de publica- tion scientifique}

$\mathrm{Au}$ point à l'ordre du jour «Révision des programmes de formation postgraduée», Werner Bauer s'est penché sur un cas rare: celui où une société de discipline a désapprouvé les décisions préalables du Comité de l'ISFM. Dans ce contexte, le plénum a dû statuer sur deux propositions de swiss orthopaedics. La première concernait la formation postgraduée de base obligatoire. Selon l'idée de la société de discipline, celle-ci devrait être facultative et pouvoir être remplacée par une année de formation postgraduée spécifique en chirurgie orthopédique. Cette proposition était incontestée et a été approuvée avec seulement deux oppositions et quatre abstentions.

La deuxième proposition qui demandait qu'une thèse de doctorat ne doive, contrairement à l'avis du Comité de l'ISFM, pas forcément être acceptée par swiss orthopaedics comme publication scientifique en vue de l'obtention du titre de spécialiste, a suscité une vive discussion. Seuls les articles ayant été soumis à un peer review devraient être pris en compte. Les deux positions ont été défendues avec verve par différents intervenants. Lors du vote, le plénum a finalement décidé par 49 voix contre 9 de maintenir la réglementation formulée par le Comité et approuvée par le plénum en 2016. Une thèse de doctorat continuera donc d'être reconnue comme publication scientifique pour le titre de spécialiste «Chirurgie orthopédique et traumatologie de l'appareil locomoteur».

La révision du programme de formation postgraduée de la Société suisse de médecine légale et l'introduction au $1^{\mathrm{er}}$ janvier 2018 d'un examen sanctionnant la formation approfondie en neuro-urologie ont été approuvés sans discussion.

\section{eccElearning: le prototype d'un concept de Blended Learning}

Dans son exposé, Max Aebi a présenté le programme en ligne eccElearning [2], une application internet pour enseigner les connaissances médicales. Durant sa carrière, ce chirurgien orthopédique maintes fois récompensé a été professeur, directeur et médecin-chef du service de chirurgie orthopédique au Centre universitaire de santé McGill. Il est co-fondateur et rédacteur en chef d'eccElearning. Ce nom désigne le premier cursus en ligne d'une sous-spécialité chirurgicale qui a été développé selon le concept de Blended Learning. Comme l'a expliqué et démontré M. Aebi, l'eccElearning intègre les phases d'apprentissage «réelles» en face-à-face et les phases "virtuelles» dans un seul environnement d'apprentissage. Le but est de réunir de

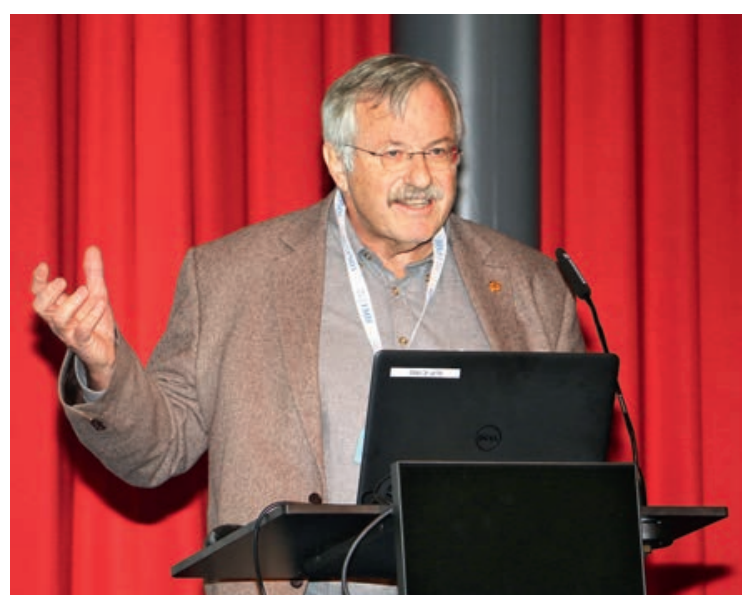

Max Aebi présente la plateforme «eccElearning" qu'il a contribué à développer.

façon optimale les avantages des deux méthodes d'enseignement et d'apprentissage. Le programme compte actuellement neuf modules comprenant 18 à 25 «cours magistraux» (lectures). Chaque module inclut aussi un Live Education Event et s'achève par un examen. A cela viennent s'ajouter 16 vidéos didactiques consacrées à la chirurgie de la colonne vertébrale et des milliers de références. Le cursus de formation est conçu sur une période de deux à trois ans et se termine par un Surgical skill assessment. De par sa structure, le programme pourrait aussi être appliqué à d'autres disciplines.

\section{Comment font les pilotes?}

L'après-midi, l'assemblée plénière s'est poursuivie avec un autre exposé passionnant, cette fois d'un point de vue non médical. Daniel Stalder, commandant de bord chez Swiss, a montré à l'aide de nombreux exemples pratiques comment les pilotes maintiennent leur niveau de connaissances. Avec huit jours de formation

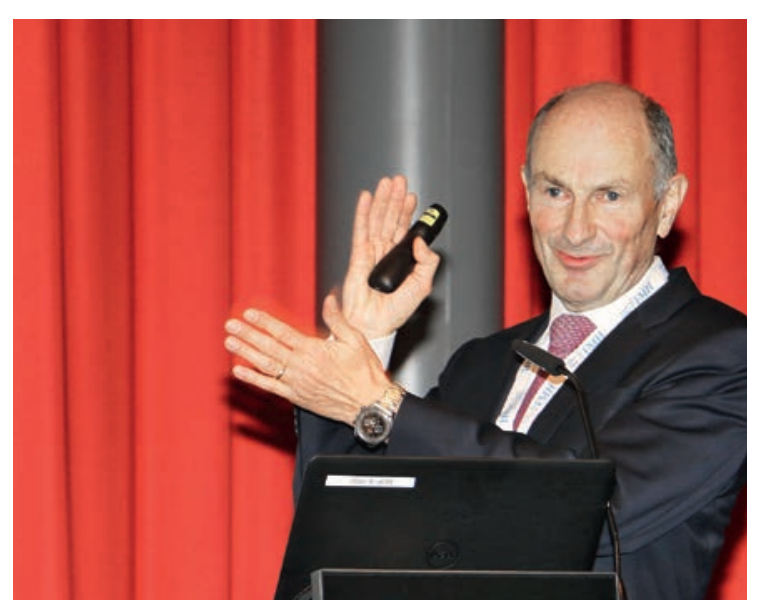

Daniel Stalder, commandant de bord chez Swiss, donne un aperçu des standards de formation des pilotes. 
continue obligatoire par année, les exigences pour les pilotes de Swiss sont élevées. Swiss se sert aussi d'applications internet, par exemple sous forme de tests en ligne, qui doivent être accomplis avant la journée de formation continue à proprement parler. L'entraînement des pilotes se caractérise par une très forte standardisation impliquant des exercices répétés de rares situations d'urgence. L'apprentissage à partir d'erreurs est fortement institutionnalisé et s'accompagne d'une prise de conscience de l'erreur et d'une transparence maximale après des incidents critiques ou des accidents. Les nombreuses questions posées à l'orateur ont témoigné du vif intérêt du public pour les explications de Daniel Stalder.

La formation continue interprofessionnelle gagne en importance. Regula Schmid, vice-présidente de l'ISFM, a relaté une manifestation consacrée à ce sujet. En comparaison internationale, la Suisse ne compte certes pas parmi les précurseurs, mais en Suisse aussi, il existe un certain nombre de projets intéressants. Les institutions comme l'OFSP ou l'Académie suisse des sciences médicales (ASSM) ont reconnu l'importance de l'interprofessionnalité et s'engagent dans ce domaine. L'oratrice a conclu que l'apprentissage interprofessionnel est utile au quotidien et qu'il contribue globalement à l'amélioration de la prise en charge médicale.

\section{Entre responsabilité individuelle et régulation}

L'organisation actuelle de la formation médicale continue, qui est un facteur important pour la qualité du système de santé, est-elle encore au goût du jour? Sommes-nous déjà surréglementés ou faut-il au contraire davantage de dispositions et de contrôles? Ou faut-il même procéder à une recertification après un certain nombre d'année d'exercice de la profession telle qu'elle est par exemple de plus en plus souvent exigée aux Etats-Unis ou en Angleterre? Dans la dernière partie du plénum, Werner Bauer a animé un débat ouvert sur ces questions. Comme l'on pouvait s'y attendre, la discussion n'a pas permis de trouver des réponses définitives. Mais même si certaines voix étaient d'avis que la formation continue relève de la responsabilité individuelle des diplômés universitaires et de leur éthique professionnelle, l'opinion générale allait dans un autre sens. Différents délégués étaient d'avis que les objectifs de formation doivent être définis plus concrètement et mis à jour, que le corps médical doit lui-même accrô̂-

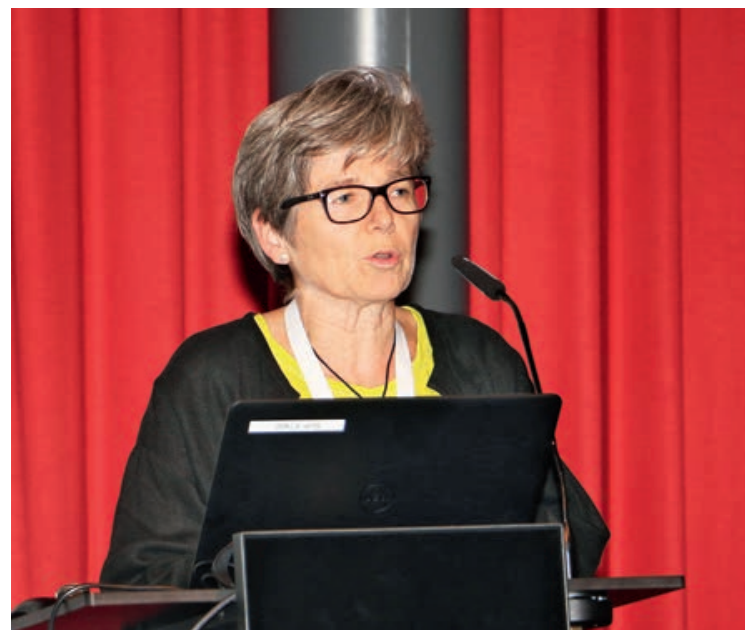

Regula Schmid relate une manifestation consacrée à la formation continue interprofessionnelle.

tre les exigences en matière de responsabilité individuelle et par exemple rendre obligatoire le diplôme de formation continue. Cet avis repose-implicitement ou explicitement - sur la conviction qu'il vaut mieux, en tant que corps médical, agir soi-même avant que la politique et les autorités ne prennent les affaires en main avec des réglementations et contrôles.

\section{Assemblée plénière de I'ISFM}

Organe autonome de la FMH, I'Institut suisse pour la formation médicale postgraduée et continue (ISFM) réunit tous les principaux acteurs et organismes du domaine de la formation médicale postgraduée et continue, et garantit aux médecins une formation de qualité élevée dans plus de 120 domaines spécialisés. L'assemblée plénière annuelle de I'ISFM remplit différentes fonctions. D'une part, les membres du plénum forment un Comité doté d'un pouvoir de décision et d'élection, qui traite les affaires relevant de son domaine de compétences à l'instar de la Chambre médicale de la FMH. De I'autre, l'assemblée, à laquelle sont également conviés des invités de provenances diverses, constitue une plate-forme chargée de transmettre des informations sur des thèmes spécifiques, de garantir l'échange d'expérience entre les participants et de débattre de toutes sortes de sujets.

\section{Crédits photo \\ Bruno Kesseli}

\section{Références}

1 Les principales nouveautés ont déjà été publiées dans le BMS dans l'article suivant: Kuhn H, Hänggeli C. La LPMéd révisée entre en vigueur au $1^{\text {er }}$ janvier 2018. Bulletin des médecins suisses 2017;98(51/52): 1727-8. https://bullmed.ch/article/doi/ saez.2017.06295/

2 Des informations détaillées au sujet d'eccElearning sont publiées sur www.eccelearning.com 